\title{
Kommentar zu Wenn Werte entscheiden
}

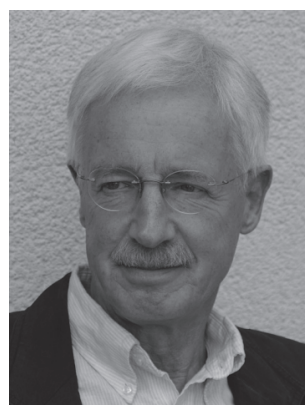

\section{Andreas Remer}

Die Frage, ob es im nennenswerten Umfang Führungskräfte gibt, die sich am Wert der „Nachhaltigkeit“ zu orientieren bereit sind, ist zweifellos von Interesse. Man muss allerdings schon gleich hinzufügen, dass auch in diesem Fall „der Geist willig und das Fleisch schwach“ sein kann. Von noch grösserer Relevanz wäre deshalb die Frage gewesen, wann und inwieweit Führungskräfte tatsächlich im Sinne von „Nachhaltigkeit“ entscheiden. Aber wie so oft heutzutage entscheidet hier die Datenbeschaffbarkeit über die Forschungsfrage. Dies muss bei den derzeitigen Qualifizierungspraktiken insbesondere jüngeren Forschern nachgesehen werden.

Die weitergehende Frage ist dann, wen und warum das Thema interessiert. Vermutlich haben die „Stakeholder“ ganz unterschiedliche Ansichten hierzu. Als eine Art gemeinsamer Nenner wird dann oftmals das Wohl des Unternehmens und, wie auch in diesem Beitrag, ein „Wettbewerbsvorteil“ postuliert. Damit drohte die Nachhaltigkeitsdebatte auf das Niveau der klassischen Erfolgsfaktoren-Forschung zurückzufallen. Das kann nicht die Absicht der Autoren gewesen sein. Den Bezug zu konkreten Erfolgskriterien wie etwa Gewinn oder Umsatz lassen sie vermutlich ganz bewusst offen. Aber wozu dann eigentlich „Nachhaltigkeit"? Die Antwort auf diese Frage setzt voraus, dass zunächst Klarheit über den Begriff geschaffen wird.

Nachhaltigkeit drückt sich hier darin aus, dass die Möglichkeit zukünftiger Bedürfnisbefriedigung aufrechterhalten wird. Als Definition ist dies wenig angreifbar, da es offen lässt, um wessen und welche Bedürfnisse es geht und was als befriedigend anzusehen ist. Soll z.B. eine „nachhaltige Entwicklung“ garantieren, dass auch künftige Generationen nach Belieben konsumieren können - oder sollen sich die Bedürfnisse auch gleich mit entwickeln? Dürfte oder müsste im Interesse der Nachhaltigkeit auch das eine oder andere Unternehmen (z.B. Atomkraft) geopfert werden, oder ist nicht doch eher der langfristige Unternehmenserfolg gemeint? Ohne einen klaren Bezugspunkt jedenfalls hängt auch der Dreiklang „ökologisch - ökonomisch - sozial“ ein wenig in der Luft. Selbst, wenn dieser Bezugspunkt nur im „Wettbewerbsvorteil“ auf dem Markt gesehen würde, kann die Ursache/Wirkungs-Beziehung keineswegs als geklärt oder gar als linear unterstellt werden. Wieso sollte ein Unternehmen, dessen Führungskräfte (siehe die drei Indikatorenlisten im Anhang des Artikels) „grün, sparsam und freundlich“ entscheiden, unter heutigen Verhältnissen einen Wettbewerbsvorteil besitzen? Es muss sich also hinter „Nachhaltigkeit“ etwas anderes verbergen als der „Erfolg“. Verräterisch ist in diesem Zusammenhang die Formel vom „nachhaltigen Erfolg“. Ein Unternehmen, das auch morgen noch Erfolg haben will, muss vor allem morgen überhaupt noch $d a$ sein. Die paradigmatischen Konsequenzen dieser einfachen Einsicht liegen auf der Hand: Statt auf Erfolg im Sinne von Zweckerreichung bezieht sich Nachhaltigkeit auf das Problem der Existenz eines Systems in einer problematischen Umwelt. Alles andere muss offen bleiben, wenn „Nachhaltigkeit“ nicht zu einem betriebswirtschaftlichen Erfolgsfaktor verkommen soll. 
Insofern erscheinen zwei Kerngedanken der Autoren als völlig berechtigt, nämlich dass, erstens, „nachhaltiges“ Entscheiden ein vieldimensionales Problemspektrum beinhaltet und dass, zweitens, die Komplexität des Problems zunehmend auf der Ebene der Person abgearbeitet werden muss. Ein aufschlussreiches Ergebnis der Clusteranalyse ist die Feststellung, dass die „Nachhaltigkeits-Gruppe“ schlichtweg alle drei Problemdimensionen für wichtiger hält als der Kontroll-Cluster. Was aber wäre der Preis, wenn dies in die Entscheidungsprozesse des Unternehmens einginge? Der ehemals eindeutige Zweck als komplexitätsreduzierende Leitlinie müsste mehr und mehr problemorientierten Erhaltungsformeln weichen, wie sie sich ja mit der „Beachtung der Stakeholderbedürfnisse“ oder dem „Resource-based View“ oder „sustainability scorecards“ bereits andeuten. Da diese Formeln aber in einem hohen Masse interpretationsfähig sind, dürfte die Versuchung immer grösser werden, die Entscheider mit den „richtigen“ Werten auszurüsten, will man nicht völlige Beliebigkeit riskieren.

Deshalb wäre es natürlich interessant zu erfahren, wie sich die Verfasser eine „Überprüfung der Werthaltungen “ bei der Bewerberauslese vorstellen. Soll allgemein danach gefragt werden, wie sensibel die künftigen Entscheider hinsichtlich der drei „Nachhaltigkeits“-Dimensionen sind? Und reichen diese drei Dimensionen überhaupt aus, wenn es um die Erhaltung des Unternehmens (oder gar der Welt) geht? Vermutlich muss hier irgendwann das Problem von der Ebene der Auslesekriterien auf die Ebene der Ausleseprozesskriterien verlagert, also z.B. die Frage gestellt werden, wer an diesem Prozess wie zu beteiligen wäre. Dies gälte dann nebenbei bemerkt auch für alle anderen Managementthemen wie Organisation oder Planung.

Bleibt als Fazit: Der Beitrag markiert den Beginn einer heissen Spur, und es ist zu hoffen, dass diese nicht in Richtung eines weiteren Personalmarketing-Ansatzes, sondern bis „zum Ende“ verfolgt wird. Dazu gehört sicherlich auch ein noch grösseres Mass an begrifflicher Präzisierung sowie ein intensiverer Blick über den Zaun. Andere Wissenschaften, namentlich die soziologische Institutions- und Systemtheorie oder auch die psychologischen Forschungen zu „Schlüsselqualifikationen“ und zum „Corporate Citizen Behaviour“ etc. halten längst Entwürfe bereit, die sehr gut zum Thema passen.

Andreas Remer, Prof. em. Dr., war Lehrstuhlinhaber für Organisations- und Managementlehre an der Universität Bayreuth. 